\title{
UNSTEADY CONVECTION FLOW OF A MAGNETOMICROPOLAR FLUID PAST A VERTICAL POROUS PLATE
}

\author{
N.C. JAIN ${ }^{*}$ \\ Department of Mathematics \\ University of Rajasthan \\ Jaipur-302004, INDIA \\ E-mail: jainnc181@rediffmail.com \\ P. GUPTA \\ Department of Mathematics \\ Anand International College of Engineering \\ Jaipur, INDIA \\ E-mail: maths_lecturer@yahoo.co.in \\ B. SHARMA \\ Department of Mathematics \\ University of Rajasthan \\ Jaipur-302004, INDIA
}

\begin{abstract}
The paper deals with an unsteady two dimensional laminar slip flow of a viscous incompressible magnetomicropolar fluid past a semi infinite porous plate embedded in a porous medium. The flow is under the influence of a transverse magnetic field and heat source/sink. The free stream velocity follows an exponentially increasing or decreasing small perturbation law. The porous surface absorbs the fluid with time varying suction velocity. Expressions are obtained for velocity and temperature fields, mean angular velocity, skin friction and the Nusselt number.
\end{abstract}

Key words: heat source/sink, magnetic field, porous medium, slip velocity, unsteady, vertical plate.

\section{Introduction}

In the literatures, extensive research has been mode on a steady/unsteady free convection heat transfer flow in a porous medium. Details of the flow of Newtonion fluids through a porous medium were given in Kokac et al. (1991), Nield and Bejan (1992). Yamamoto and Yoshidha (1976) considered a flow with convection acceleration on a plane porous wall specifically for the flow outside the vortex layer. Chawala and Singh (1979), Raptis et al. (1981) studied flows through a porous medium considering generalized Darcy's law.

In all the above-mentioned research papers generalized Darcy's law is derived without taking into account the angular velocity of the fluid particles. Aero et al. (1965) derived and solved the flow equations of the fluid in which angular velocity of the fluid particles was considered. These fluids are known as polar fluids in the literature and are more general than ordinary fluids (Lukaszewicz, 1999). Micropolar fluids are fluids with microstructure and belong to a class of fluids with asymmetrical stress tensor. Physically, they

\footnotetext{
* To whom correspondence should be addressed
} 
represent fluids consisting of randomly oriented particles suspended in a viscous medium. Ahmedi (1976) studied the flow of a micropolar fluid. Recently, Kim (2001) studied the basic problem of a viscous incompressible micropolar fluid with fluctuating stream velocity which was also studied by Stokes (1851) for a Newtonion fluid. Because of their practical importance the unsteady flow problems were studied extensively by a number of researchers. Soundelgeker (1973) studied the time dependent flow problems on an infinite vertical plate, when it is cooled or heated by free convection currents. El Arabawy and Hassen (2003) studied the effects of suction/injection on a flow of a micropolar fluid past a continuously moving plate in the presence of radiation. Chu et al. (2002) solved the problems of micropolar fluid flows along a vertical wavy surface with a discontinuous temperature profile. Perhaps the most widely known aspects of non equilibrium gas flow in the fact that the velocity of a gas close to a surface describing slip accurately, however is still an active area of research.

In this paper it is proposed to study an unsteady two dimensional laminar slip flow of a viscous incompressible magnetomicropolar fluid past a semi infinite porous plate embedded in a porous medium. We consider the free stream to consist of a mean velocity and temperature with a superimposed exponentially small variation with time.

\section{Formulation of the problem}

Consider an unsteady two dimensional slip flow of a laminar, incompressible, magnetomicropolar fluid past a semi infinite porous plate embedded in a porous medium and subjected to the presence of an applied pressure gradient. The permeability of the porous medium is assumed as constant.

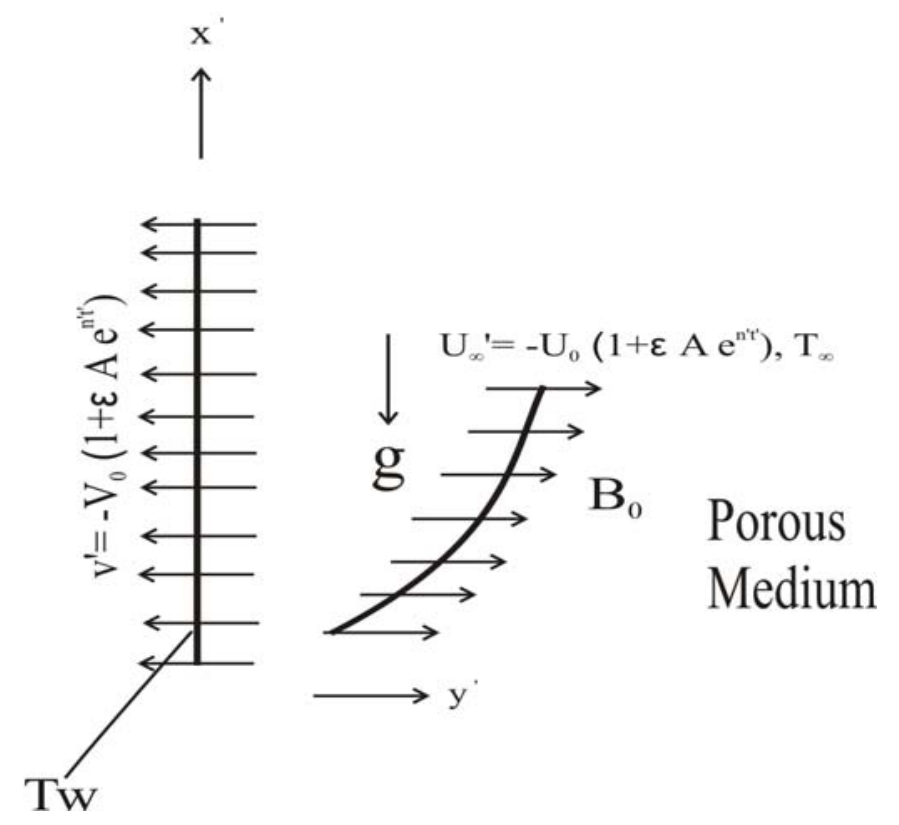

Fig. Physical model and coordinate system of the problem

Under the assumptions the flow field is governed by the following equations

$$
\begin{aligned}
& \frac{\partial v^{\prime}}{\partial y^{\prime}}=0 \\
& \frac{\partial u^{\prime}}{\partial t^{\prime}}+v^{\prime} \frac{\partial u^{\prime}}{\partial y^{\prime}}=-\frac{1}{\rho} \frac{\partial p^{\prime}}{\partial x^{\prime}}+g \beta_{f}\left(T-T_{\infty}\right)+\left(v+v_{r}\right) \frac{\partial^{2} u^{\prime}}{\partial y^{\prime 2}}-\frac{v}{K^{\prime}} u^{\prime}+2 v_{r} \frac{\partial \omega^{\prime}}{\partial y}-\frac{\sigma B_{0}^{2} u^{\prime}}{\rho}
\end{aligned}
$$




$$
\begin{aligned}
& \frac{\partial \omega^{\prime}}{\partial t^{\prime}}+v^{\prime} \frac{\partial \omega^{\prime}}{\partial y^{\prime}}=\frac{\gamma}{\rho j^{\prime}}\left(\frac{\partial^{2} \omega^{\prime}}{\partial y^{\prime 2}}\right), \\
& \frac{\partial T^{\prime}}{\partial t^{\prime}}+v^{\prime} \frac{\partial T^{\prime}}{\partial y^{\prime}}=\alpha \frac{\partial^{2} T^{\prime}}{\partial y^{\prime 2}}+\frac{S^{\prime}}{\rho C_{\rho}}\left(T-T_{\infty}\right),
\end{aligned}
$$

$x^{\prime}$ is the dimensional distance along the plate and $y^{\prime}$ is normal to the plate, $u^{\prime}$ and $v^{\prime}$ are the dimensional velocity components along $x^{\prime}$ and $y^{\prime}$, respectively, $\rho$ is the density, $v$ is the kinematic viscosity, $v_{r}$ is the kinematic rotational viscosity, $\beta_{f}$ is the coefficient of volumetric expansion of the working fluid, $K^{\prime}$ is an empirical constant called permeability of the porous medium, $j^{\prime}$ is the micro inertia density, $\omega$ is the component of angular velocity vector normal to the $x y$-plane, $\gamma$ is the spin gradient viscosity, $S$ is the heat source parameter, $B_{0}$ is the magnetic field, $T$ is the temperature, and $\alpha$ is the fluid thermal diffusivity.

The boundary conditions are as follows

$$
\begin{array}{lll}
y^{\prime}=0 ; & u^{\prime}=U_{0}+L_{1} \frac{\partial u^{\prime}}{\partial y^{\prime}}, \quad T=T_{w}+\varepsilon\left(T_{w}-T_{\infty}\right) e^{n^{\prime} t^{\prime}}, & \omega^{\prime}=-\frac{1}{2} \frac{\partial u^{\prime}}{\partial y^{\prime}}, \\
y^{\prime} \rightarrow \infty ; & u^{\prime} \rightarrow U_{\infty}^{\prime}=U_{0}\left(1+\varepsilon e^{n^{\prime} t^{\prime}}\right), \quad T^{\prime} \rightarrow T_{\infty}, & \omega^{\prime} \rightarrow 0 .
\end{array}
$$

Equation of continuity Eq.(2.1), shows that the suction velocity normal to the plate is a function of time only and we shall take it in the form

$$
v^{\prime}=-V_{0}\left(1+\varepsilon A e^{n^{\prime} t^{\prime}}\right)
$$

where $A$ is any real constant, $A \varepsilon<<1$ and there is small variation with time. $V_{0}$ is the scale of suction velocity which has a non zero positive constant.

Outside the boundary layer, Eq.(2.2) gives

$$
-\frac{1}{\rho} \frac{\partial p^{\prime}}{\partial x^{\prime}}=\frac{d U_{\infty}^{\prime}}{d t^{\prime}}+\frac{v}{K^{\prime}} U_{\infty}^{\prime}+\frac{\sigma}{\rho} B_{0}{ }^{2} U_{\infty}^{\prime} .
$$

Now, we introduce the dimensionless quantities as

$$
\begin{aligned}
& y=\frac{y^{\prime} V_{0}}{v}, \quad t=\frac{V_{0}^{2} t^{\prime}}{v}, \quad M=\frac{\sigma B_{0}^{2} v}{\rho V_{0}^{2}}, \quad u=\frac{u^{\prime}}{U_{0}}, \quad v=\frac{v^{\prime}}{V_{0}}, \quad K=\frac{K^{\prime} V_{0}^{2}}{v^{2}} \\
& \theta=\frac{T-T_{\infty}}{T_{w}-T_{\infty}}, \quad U_{\infty}=\frac{U_{\infty}^{\prime}}{U_{0}}, \quad \omega=\frac{v}{U_{0} V_{0}} \omega^{\prime}, \quad \beta=\frac{v_{r}}{v}, \quad \operatorname{Pr}=\frac{v \rho C_{p}}{\kappa}=\frac{v}{\alpha}, \\
& n=\frac{n^{\prime} v}{V_{0}^{2}}, \quad G=\frac{v g \beta_{f}\left(T_{w}-T_{\infty}\right)}{U_{0} V_{0}^{2}}, \quad j=\frac{V_{0}^{2}}{v^{2}} j^{\prime}, \quad h=\frac{L_{1} V_{0}}{v}, \quad S=\frac{S^{\prime} v}{\rho C_{p} V_{0}^{2}},
\end{aligned}
$$


and the spin gradient viscosity $\gamma$ is defined as

$$
\gamma=\left(\mu+\frac{\wedge}{2}\right) j^{\prime}=\mu j^{\prime}\left(1+\frac{1}{2} \beta^{\prime}\right)
$$

where $\beta^{\prime}=\frac{\wedge}{\mu}$ is the dimensionless viscosity ratio and $\wedge$ is the coefficient of gyro viscosity (or vortex viscosity) management.

In view of Eqs (2.6) and (2.7), the governing Eq.(2.2) to Eq.(2.4) reduce to the following nondimensional form

$$
\begin{aligned}
& \frac{\partial u}{\partial t}-\left(1+A \varepsilon e^{n t}\right) \frac{\partial u}{\partial y}=\frac{d U_{\infty}}{d t}+\frac{1}{K}\left(U_{\infty}-u\right)+(1+\beta) \frac{\partial^{2} u}{\partial y^{2}}+G \theta+2 \beta \frac{\partial \omega}{\partial y}-M\left(u-U_{\infty}\right) \\
& \frac{\partial \omega}{\partial t}-\left(1+A \varepsilon e^{n t}\right) \frac{\partial \omega}{\partial y}=\frac{1}{\eta} \frac{\partial^{2} \omega}{\partial y^{2}} \\
& \frac{\partial \theta}{\partial t}-\left(1+A \varepsilon e^{n t}\right) \frac{\partial \theta}{\partial y}=\frac{1}{\operatorname{Pr}} \frac{\partial^{2} \theta}{\partial y^{2}}+S \theta
\end{aligned}
$$

with the corresponding boundary conditions

$$
\begin{array}{ll}
y=0 ; & u=1+h \frac{\partial u}{\partial y}, \quad \theta=1+\varepsilon e^{n t}, \quad \omega=-\frac{1}{2} \frac{\partial u}{\partial y}, \\
y \rightarrow \infty ; & u \rightarrow U_{\infty}=1+\varepsilon e^{n t}, \quad \omega \rightarrow 0, \quad \theta \rightarrow 0
\end{array}
$$

where

$$
\eta=\frac{\mu j^{\prime}}{\gamma}=\left(\frac{2}{2+\beta^{\prime}}\right)
$$

\section{Solution of the problem}

To solve the partial differential Eqs (2.9) to (2.11), we assume for small $\varepsilon$

$$
f(y, t)=f_{0}(y)+\varepsilon e^{n t} f_{l}(y)+\ldots
$$

where $f$ stands for $u, \omega$ and $\theta$.

Substituting expressions (3.1) in Eqs (2.8) to (2.10) and equating the harmonic and non harmonic terms and neglecting the coefficient of $o\left(\varepsilon^{2}\right)$, we get the following sets of ordinary differential equations. 


$$
\begin{aligned}
& (1+\beta) u_{0}^{\prime \prime}+u_{0}^{\prime}-\left(\frac{1}{K}+M\right) u_{0}=-G \theta_{0}-\left(\frac{1}{K}+M\right)+2 \beta \omega_{0}^{\prime}, \\
& (1+\beta) u_{1}^{\prime \prime}+u_{1}^{\prime}-\left(\frac{1}{K}+M+n\right) u_{1}=-G \theta_{1}-\left(\frac{1}{K}+M\right)+2 \beta \omega_{1}^{\prime}-A u_{0}^{\prime}, \\
& \omega_{0}^{\prime \prime}+\eta \omega_{0}^{\prime}=0, \\
& \omega_{1}^{\prime \prime}+\eta \omega_{1}^{\prime}-n \eta \omega_{1}=-A \eta \omega_{0}^{\prime}, \\
& \theta_{0}^{\prime \prime}+\operatorname{Pr} \theta_{0}+S \operatorname{Pr} \theta_{0}=0, \\
& \theta_{1}^{\prime \prime}+\operatorname{Pr} \theta_{1}-(n+S) \operatorname{Pr} \theta_{1}=-A \operatorname{Pr} \theta_{0}^{\prime} .
\end{aligned}
$$

The relevant boundary conditions are given as

$$
\begin{aligned}
& u_{0}=1+h u_{0}^{\prime}, \quad u_{1}=h u_{1}^{\prime}, \quad \theta_{0}=1, \quad \theta_{1}=1, \quad \omega_{0}=-\frac{1}{2} u_{0}^{\prime}, \quad \omega_{1}=-\frac{1}{2} u_{1}^{\prime} \quad \text { at } y=0, \\
& u_{0} \rightarrow 1, \quad u_{1} \rightarrow 1, \quad \theta_{0} \rightarrow 0, \quad \theta_{1} \rightarrow 0, \quad \omega_{0} \rightarrow 0, \quad \omega_{1} \rightarrow 0 \quad \text { as } \quad y \rightarrow \infty .
\end{aligned}
$$

The prime denotes differentiation with respect to $y$. results

On solving Eqs (3.2) to (3.7) with the help of boundary conditions (3.8), we get the following

$$
\begin{aligned}
& u_{0}=C_{5} e^{-m_{7} y}-D_{3} e^{-m_{3} y}-D_{4} C_{1} e^{-\eta y}+D_{5}, \\
& u_{1}=C_{6} e^{-m_{9} y}+D_{6} e^{-m_{7} y}+D_{7} e^{-m_{3} y}+D_{8} e^{-\eta y}-D_{9} e^{-m_{5} y}+D_{10} C_{2} e^{-m_{1} y}+D_{11}, \\
& \theta_{0}=C_{3} e^{-m_{3} y}, \\
& \theta_{1}=C_{4} e^{-m_{5} y}-D_{2} e^{-m_{3} y}, \\
& \omega_{0}=C_{1} e^{-\eta y}, \\
& \omega_{1}=C_{2} e^{-m_{1} y}-D_{1} e^{-\eta y}
\end{aligned}
$$

where

$$
m_{1}=\frac{\eta+\sqrt{\eta^{2}+4 n \eta}}{2}, \quad m_{2}=\frac{-\eta+\sqrt{\eta^{2}+4 n \eta}}{2}
$$




$$
\begin{aligned}
& m_{3}=\frac{\operatorname{Pr}+\sqrt{\operatorname{Pr}^{2}-4 \operatorname{Pr} S}}{2}, \quad m_{4}=\frac{-\operatorname{Pr}+\sqrt{\operatorname{Pr}^{2}-4 \operatorname{Pr} S}}{2}, \\
& m_{5}=\frac{\operatorname{Pr}+\sqrt{\operatorname{Pr}^{2}+4 \operatorname{Pr}(n+S)}}{2}, \quad m_{6}=\frac{-\operatorname{Pr}+\sqrt{\operatorname{Pr}^{2}+4 \operatorname{Pr}(n+S)}}{2}, \\
& m_{7}=\frac{1+\sqrt{1+4(1+\beta)\left(\frac{1}{K}+M\right)}}{2}, \quad m_{8}=\frac{-1+\sqrt{1+4(1+\beta)\left(\frac{1}{K}+M\right)}}{2}, \\
& D_{1}=\frac{A \eta C_{1}}{n}, \quad D_{2}=\frac{A \operatorname{Pr} m_{3}}{m_{3}^{2}-m_{3} \operatorname{Pr}-\operatorname{Pr}(n+S)}, \quad D_{3}=\frac{\mathrm{Gr}}{(1+\beta) m_{3}^{2}-m_{3}-\left(\frac{1}{K}+M\right)}, \\
& D_{4}=\frac{2 \eta \beta}{\left\{(1+\beta) \eta^{2}-\eta-\left(\frac{1}{K}+M\right)\right\}}, \quad D_{5}=1, \\
& D_{6}=\frac{A C_{5} m_{7}}{(1+\beta) m_{7}^{2}-m_{7}-\left(\frac{1}{K}+M+n\right)}, \quad D_{7}=\frac{\left(\mathrm{Gr} D_{2}-A D_{3} m_{3}\right)}{(1+\beta) m_{3}^{2}-m_{3}-\left(\frac{1}{K}+M+n\right)}, \\
& D_{8}=\frac{\left(A D_{4} C_{1} \eta-2 \beta D_{1} \eta\right)}{(1+\beta) \eta^{2}-\eta-\left(\frac{1}{K}+M^{2}+n\right)}, \quad D_{9}=\frac{\mathrm{Gr}_{4}}{(1+\beta) m_{5}^{2}-m_{5}-P\left(\frac{1}{K}+M+n\right)}, \\
& D_{10}=\frac{2 \beta m_{1}}{(1+\beta) m_{1}^{2}-m_{1}-\left(\frac{1}{K}+M+n\right)}, \quad D_{11}=\frac{\left(\frac{1}{K}+n\right)}{\left(\frac{1}{K}+M+n\right)}, \\
& K_{1}=D_{4}+h \eta D_{4}, \quad K_{2}=1+h m_{7}, \quad K_{3}=1+h D_{3} m_{3}+D_{3}-1, \\
& K_{4}=1+\frac{\eta D_{4}}{2}, \quad K_{5}=\frac{m_{7}}{2}, \quad K_{6}=\frac{-D_{3} m_{3}}{2}, \quad K_{7}=1-\frac{D_{10} m_{1}}{2} \\
& K_{8}=\frac{m_{9}}{2}, \quad K_{9}=D_{1}+\frac{1}{2}\left[D_{6} m_{7}+D_{7} m_{3}+D_{8} \eta-D_{9} m_{5}\right] \\
& K_{10}=D_{10}+h m_{1} D_{10}, \quad K_{11}=1+h m_{9}
\end{aligned}
$$




$$
\begin{aligned}
& C_{1}=\frac{K_{3} K_{5}+K_{2} K_{6}}{K_{1} K_{5}+K_{2} K_{4}}, \quad C_{2}=\frac{K_{12} K_{8}+K_{9} K_{11}}{K_{10} K_{8}+K_{7} K_{11}}, \\
& C_{3}=1, \quad C_{4}=1+D_{2} C_{2}=\frac{K_{12} K_{8}+K_{9} K_{11}}{K_{10} K_{8}+K_{7} K_{11}} .
\end{aligned}
$$

Using Eq.(3.1), we obtain the main, angular velocity and temperature as

$$
\begin{aligned}
& u(y, t)=C_{5} e^{-m_{7} y}-D_{3} e^{-m_{3} y}-D_{4} C_{1} e^{-\eta y}+1+ \\
& +\varepsilon e^{n t}\left(C_{6} e^{-m_{9} y}+D_{6} e^{-m_{7} y}+D_{7} e^{-m_{3} y}+D_{8} e^{-\eta y}-D_{9} e^{-m_{5} y}+D_{10} C_{2} e^{-m_{1} y}+D_{11}\right), \\
& \omega(y, t)=\left(C_{1} e^{-\eta y}\right)+\varepsilon e^{n t}\left(C_{2} e^{-m_{1} y}-D_{1} e^{-\eta y}\right), \\
& \theta(y, t)=\left(C_{3} e^{-m_{3}^{y}}\right)+\varepsilon e^{n t}\left(C_{4} e^{-m_{5} y}-D_{2} e^{-m_{3}^{y}}\right) .
\end{aligned}
$$

\section{Skin friction and Nusselt number} obtained as

After obtaining velocity distribution, the important parameter of skin friction at the plate can now be

$$
\begin{aligned}
& \tau_{w}=\frac{\tau^{*}{ }_{w}}{\rho V_{0} U_{0}}=\left[\frac{\partial u}{\partial y}\right]_{y=0}=\left\{-C_{5} m_{7}+D_{3} m_{3}-D_{4} C_{1} \eta\right\}+ \\
& +\varepsilon e^{n t}\left\{-C_{6} m_{9}-D_{6} m_{7}-D_{7} m_{3}-D_{8} \eta+D_{9} m_{5}-D_{10} C_{2} m_{1}\right\} .
\end{aligned}
$$

From Eq.(3.17), we calculate the rate of heat transfer in terms of the Nusselt number as

$$
\mathrm{Nu}=x \frac{\left(\partial T / \partial y^{*}\right)_{w}}{T_{w}-T_{\infty}}=\left[\frac{\partial \theta}{\partial y}\right]_{y=0}=\left\{-C_{3} m_{3}\right\}+\varepsilon e^{n t}\left\{m_{5} C_{4}+m_{3} D_{2}\right\}
$$

\section{Discussions and conclusion}

The effects of suction velocity varying exponentially in time about a non zero constant mean value on the flow and heat transfer of an incompressible magnetomicropolar fluid along a vertical porous plate were determined for different values of $\beta$ (rotational parameter), $K$ (permeability parameter), $S$ (heat source parameter), $h$ (slip parameter) and $\mathrm{Gr}$ (Grashof number) fixing $\varepsilon=0.1, \eta=0.1, A=0.5, n=0.1, t=1$.

In Fig.1, the velocity distribution is plotted against $y$ taking air $(\mathrm{Pr}=0.71)$ as a fluid. It is observed that the velocity increases with the increase of $h, S, M$, and $K$, but when the rotational parameter is increased the velocity has decreases.

Moreover, the velocity increases for an increase in Gr but a fall in velocity is observed for negative values of Gr. We may deduce that these results are consistent with the physical observations for the case of cooling or heating of the surface by natural convection.

The variation of temperature against $y$ is illustrated in Fig.2. Here, it is interesting to note that for the case of air an increase in the source parameter increases the temperature but for water the temperature decreases with an increase of the source parameter. 
$\operatorname{Pr}=0.71, \mathrm{n}=0.1, \mathrm{t}=1.0, \mathrm{~A}=0.5, \eta=0.1, \epsilon=0.1$,

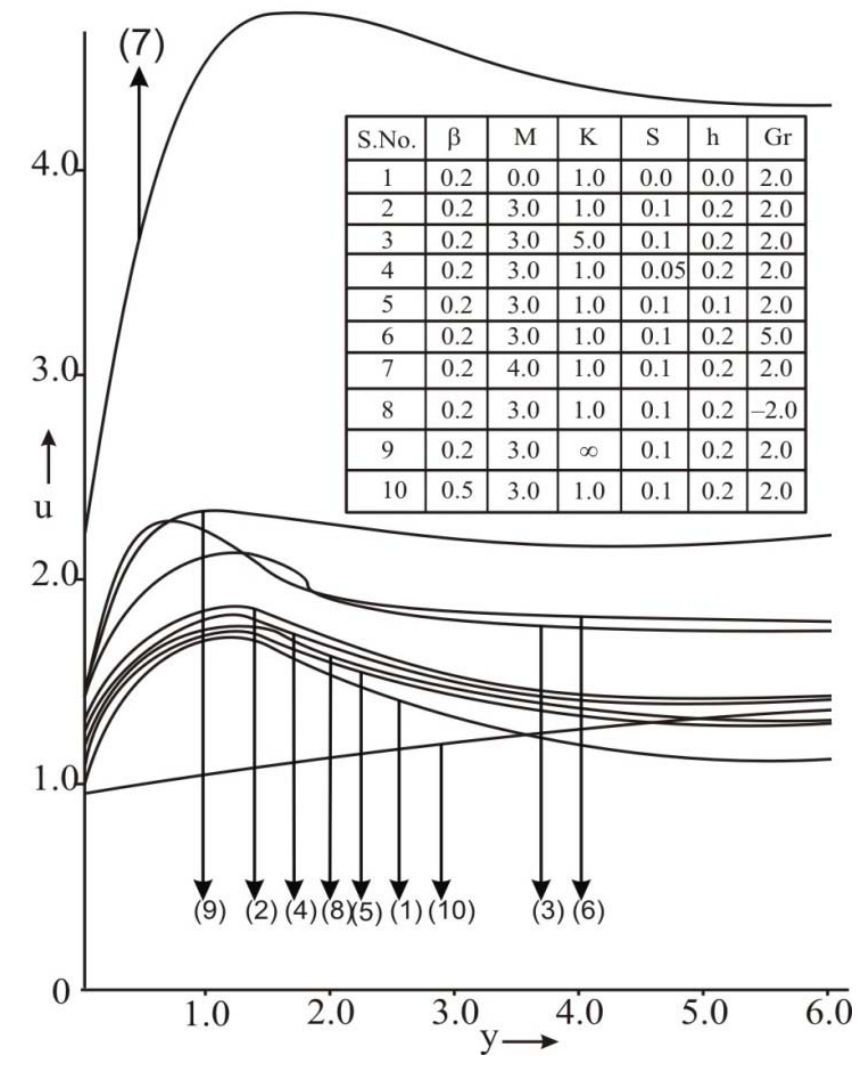

Fig.1.Velocity distribution plotted against $y$ for different values of $\beta, M, K, S, h$ and $\mathrm{Gr}$.

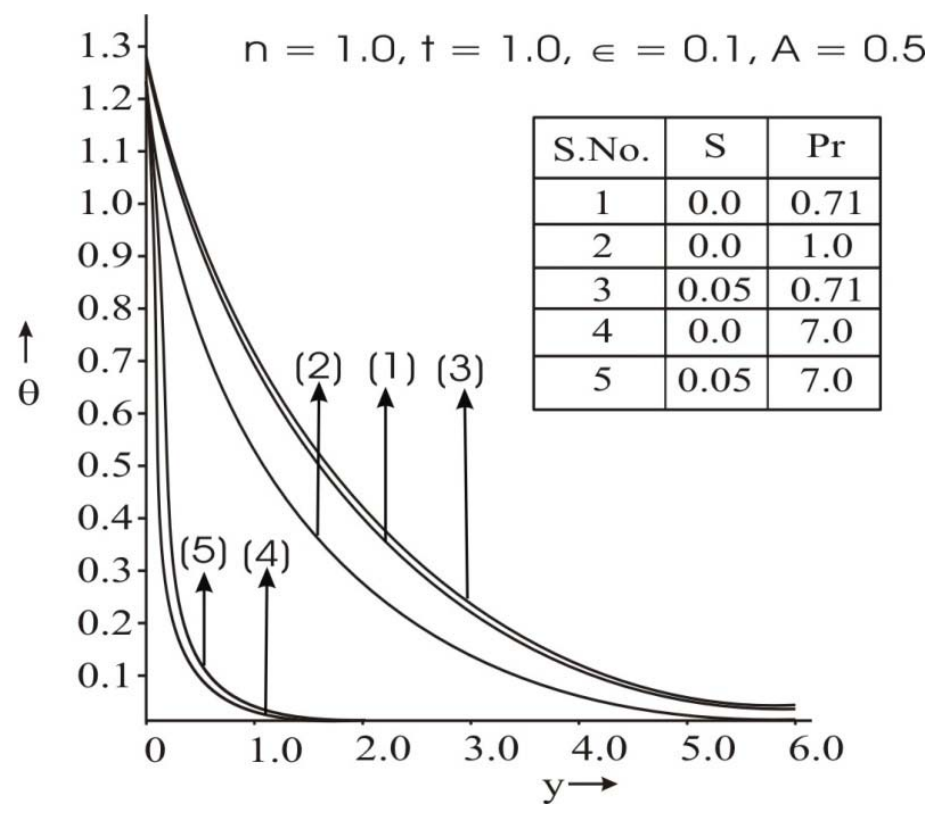

Fig.2. Temperature distribution plotted against $y$ for different values of $S$ and $\operatorname{Pr}$. 
Moreover, an increase in Pr decreases the temperature of the fluid. The reason is that smaller values of the Prandtl number are equivalent to increasing thermal conductivity and therefore heat is able to diffuse away from the heated surface more rapidly.

In Fig.3, the non dimensional angular velocity vector is plotted for different values of $S$ and Gr.

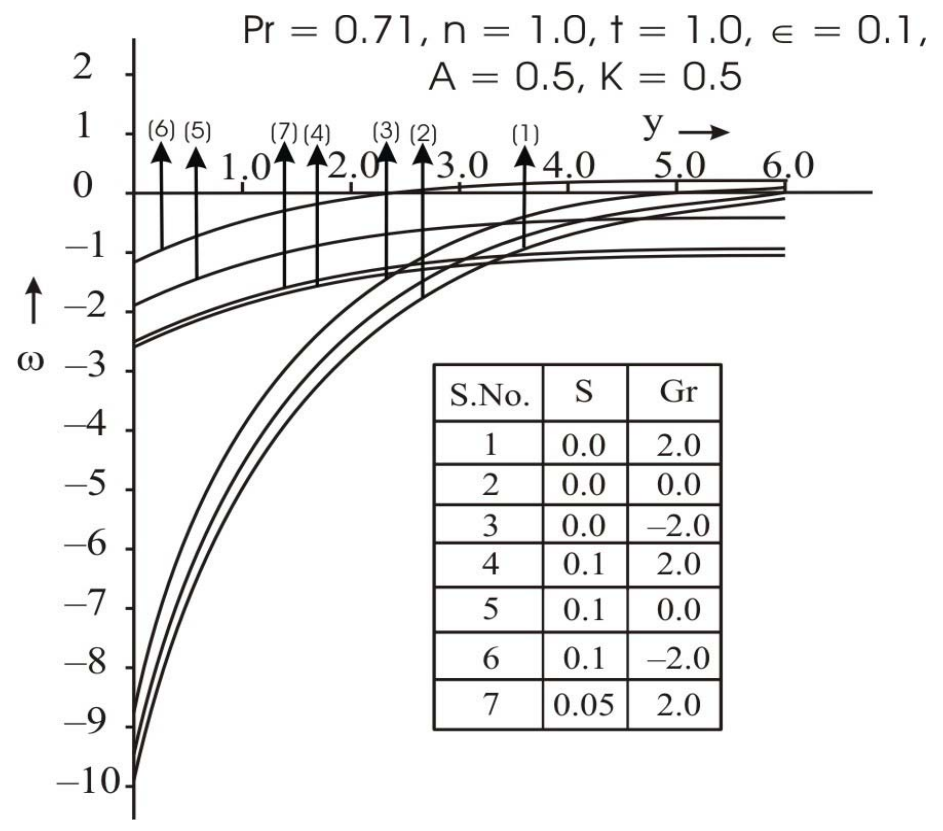

Fig.3. Angular velocity $\omega$ plotted against $y$ for different values of $S$ and Gr.

It is noted that angular velocity decreases with the increase of $S$ and Gr.

The skin friction $\tau$ is plotted against $\beta$ at the cooling plate in Fig.4.

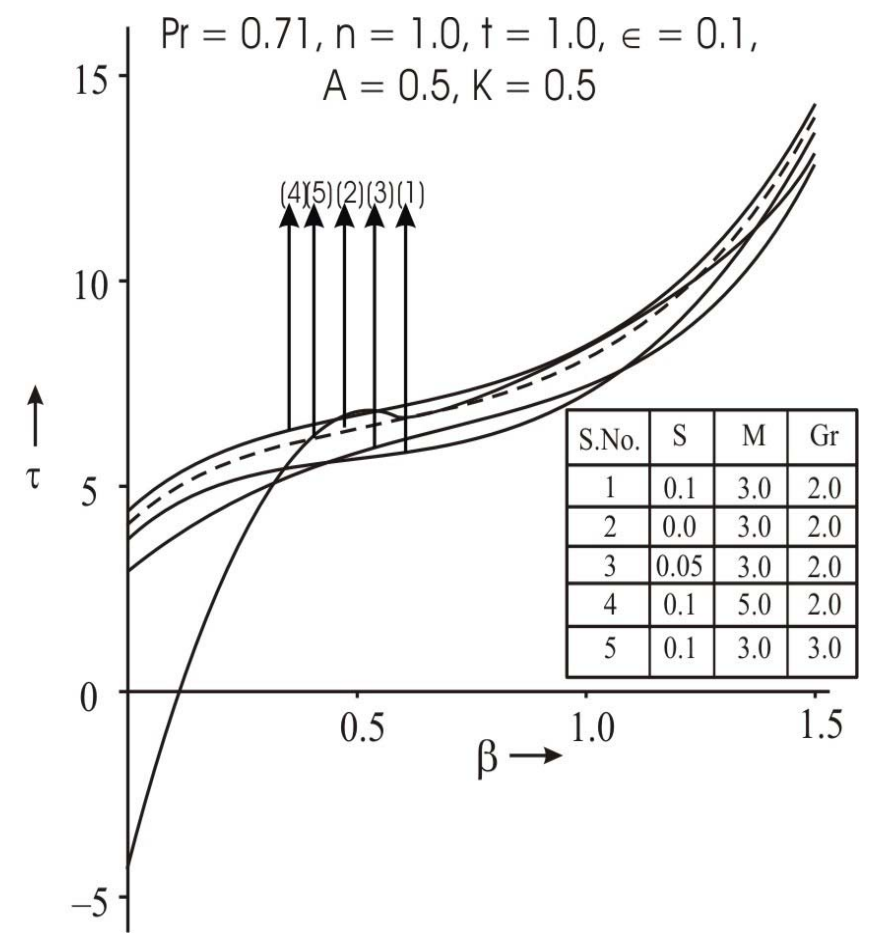

Fig.4. Skin friction ( $\tau$ ) plotted against $\beta$ for different values of $S, M$ and $\mathrm{Gr}$. 
It is observed that an increase in $M$ and $\mathrm{Gr}$ increases $\tau$, but an increase in $S$ decreases the skin friction. For the Nusselt number $(\mathrm{Nu})$ at the plate $y=0$, we observed from Fig.5, that it increases with the increase of $S$, and decreases with the increase of Pr.

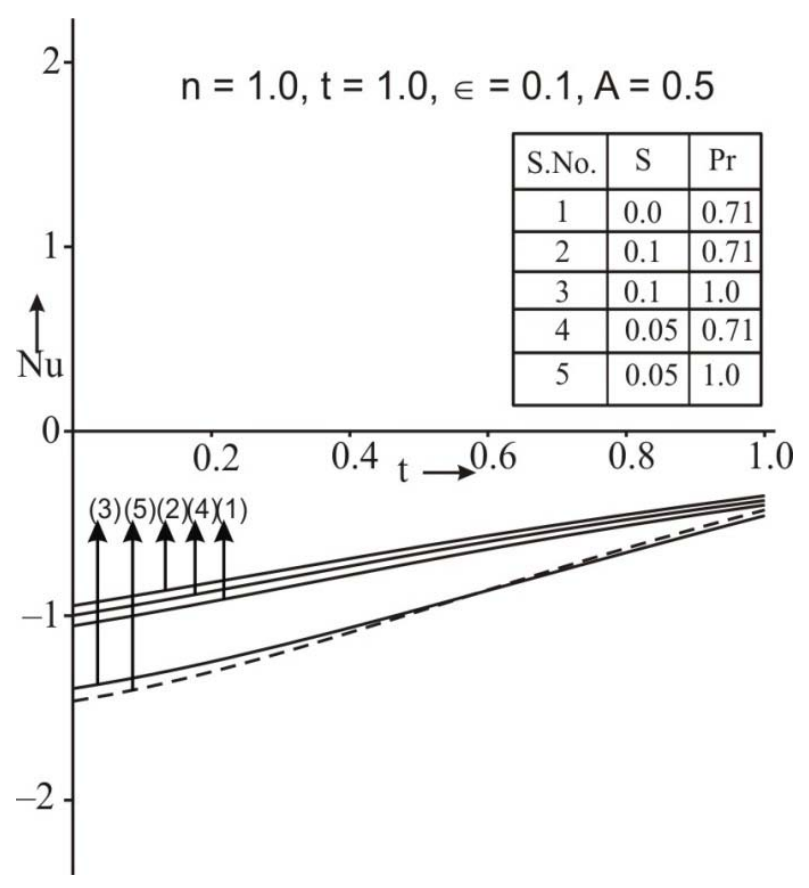

Fig.5. Nusselt Number Nu plotted against $t$ for different values of $S$ and Pr.

\section{Acknowledgements}

One of the authors (PG) is grateful to Prof (Dr.) Anurag Misra, Director, Anand International College of Engineering, Jaipur for constant encouragement during the preparation of the paper.

\section{Nomenclature}

$A$ - suction velocity amplitude

$B_{0}-$ magnetic field

$C_{p}$ - specific heat at constant pressure

$E$ - scalar constant $(<<1)$

Gr - Grashof number

$g$ - acceleration due to gravity

$K$ - permeability of the porous medium

$\mathrm{Nu}$ - Nusselt number

$n$-dimensionless exponential index

Pr - Prandtl number

$S$ - heat source parameter

$T$ - temperature

$t$-dimensionless time

$U_{0}-$ scale of free stream velocity

$u, v$ - component of velocity along and perpendicular to the plate, respectively

$V_{0}-$ scale of suction velocity 


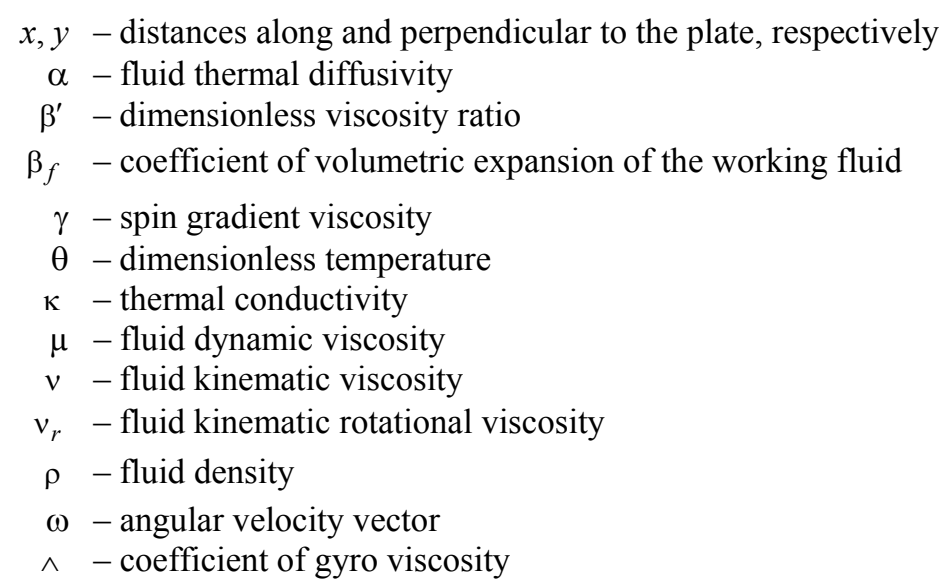

\section{References}

Aero E. L., Bulgyin A.N. and Kuvshinsky E.V. (1965): Asymmetric hydrodynamics. - J. Appl. Math. Mech., vol.29, pp.333-346.

Ahmadi G. (1976): Self similar solution of incompressible micropolar boundary layer flow over a semi infinite plate. Int. J. Engg. Science, vol.14, pp.639-646.

Chawala S.S. and Singh S. (1979): Oscillatory flow past a porous bed. - Acta Mechanica, vol.34(3-4), pp.205-213.

Chu Y.H., Hsu P.T., Liu Y.H. and Chiu L.H. (2002): Mixed convection of micropolar fluids along a vertical wavy surface with a discontinuous temperature profile. - Numerical Heat Transfer Applications; part A, vol.42, pp.733755.

El. Arabawy and Hassan A.M. (2003): Effects of suction/ injection on the flow of a micro polar fluid past a continuously moving plate in the presence of radiation. - Int. J. Heat and Mass Transfer, vol.46(8), pp.1471-1477.

Kim Y.J. (2001): Unsteady convection flow of micropolar fluids past a vertical porous plate embedded in a porous medium. - Acta Mechanica, vol.148, pp.105-116.

Kokac et al. (1991): Convective Heat and Mass Transfer in Porous Media. - Netherlands: Kluwer Academic Publisher.

Łukaszewicz G. (1999): Micropolar Fluids. - Theory and Applications, Boston, Birkhauser.

Nield D.A. and Bejan A. (1992): Convection in Porous Media. - New York: Springer.

Raptis A., Perdikis C.P. and Tzivanidis G. (1981): Free convection flow through a porous medium bounded by a vertical surface. - J. Phys. D. Appl. Phys.,vol.14, L99-L102.

Soundelgeker V.M. (1973): Free convection effects of the oscillatory flow past an infinite, vertical porous plate with constant suction. - Proc. Roy. Soc., London, A333, pp.25-36.

Stokes G.G. (1851): On the effect of the internal friction of fluids on the motion of pendulums. - Trans. Camb. Phil. Soc., vol.9, pp.8-106.

Yamamoto K. and Iwamura N. (1976): Flow with convective acceleration through a porous medium. - J. Engg. Math., vol.10, pp.41-54.

Received: November 14, 2011

Revised: July 3, 2012 